\title{
Developments in an industry-led R\&D program for recycling PVC products in Japan
}

\author{
Shigetaka Seki $\cdot$ Fumio Osakada $\cdot$ Toshiaki Yoshioka
}

Received: 5 September 2013/Accepted: 2 December 2013/Published online: 4 April 2014

(C) The Author(s) 2014. This article is published with open access at Springerlink.com

\begin{abstract}
To facilitate recycling, not only R\&D but also the creation of efficient recycling systems-collection and processing of recyclates as well as finding appropriate enduses-is essentially important. PVC has a particular advantage in regards to mechanical recycling when compared to other major plastics. However, there is no onesize-fits-all approach to a solution. Specific situations of used or off-cut products and their applications need to be well studied. Vinyl industry in Japan has devoted itself for many years to the promotion of recycling of vinyl products. It started an $R \& D$ support program in 2007 and seven projects have been completed since then. Some of the new developments include mechanical recycling for products like wall covering and tarpaulin which were considered difficult to recycle in the past. Progress in chemical recycling has been made with a technology for the removal of chlorine from shredder dusts. Recovery of chlorine is a challenge for the future. Collaboration among the various players involved in the recycling of a specific product is essential for obtaining a successful result.
\end{abstract}

Keywords PVC - Recycling - R\&D - Industry initiative * Sustainability

S. Seki $(\bowtie) \cdot$ F. Osakada

The Vinyl Environmental Council (VEC), 8th Floor, Rokko Building, 1-4-1 Shinkawa, Chuo-ku, Tokyo 104-0033, Japan e-mail: seki@vec.gr.jp

T. Yoshioka

Graduate School of Environmental Studies, Tohoku University,

Aramaki Aza Aoba 6-6-07, Aoba-ku Sendai 980-8579, Japan

\section{Introduction}

PVC products have an advantage over other plastics with regard to their suitability for mechanical recycling. The portion of PVC products-including both used, off-cut products, and wastes in the production facilities-recycled was estimated at $32 \%$, while in all other major plastics it was $22 \%$ in Japan (2011) [1]. In general, PVC products are used for infrastructure, housing and building supplies and are used for much longer periods than other plastic products. Long life and easiness to recycle, in addition to less use of fossil resources as compared to other major plastics, can contribute much to enhance environmental protection and sustainability. Nevertheless, there still remains a negative image which is mostly due to the past misunderstanding of association with dioxin emissions, which has been proved to be reduced dramatically by controlling incinerating conditions $[2,3]$ and not correlated to the chlorine content of the burned substances [4].

With a view to demonstrating the environmental advantages of PVC products, the PVC industry in Japan has been working on recycling PVC products. It reiterated its commitment to the promotion of recycling in 2007 and released the "PVC Recycle Vision" [5]. Similar industryled actions to facilitate PVC recycling can be found in many parts in the world. An example of such industry-wide systematic approaches to cover various types of PVC products was an initiative in Europe, or the "Vinyl 2010" $[6,7]$ and its following program of "Vinyl Plus".

In Japan, PVC resin manufacturers have been playing a leading role in developing new technological alternatives for PVC products and started an R\&D support program [8] to explore new technologies with high commercial application potential. The program covers half of the total expenses of adopted projects excluding labor costs and overheads, up to 
$20,000,000$ yen (approximately 200,000 USD in 2013) per project for 2 years.

The R\&D support program in Japan is a unique approach, although, development of new or more efficient technologies or applications for used PVC materials is a common interest for vinyl industry in many parts in the world. The "Vinyl Plus" of Europe declares that it targets for "new recycling technologies to account for 100,000 tons/year of its overall recycling target. With the support of the upstream industry, Vinyl Plus will investigate new ways to create controlled-loop solutions for difficult-to-recycle PVC material streams such as composite and/or contaminated/difficult-to-sort material. Vinyl Plus will encourage ideas and investments in new technology or expansion of existing innovative recycling solutions" [9]. The details of its approach have not been reported yet, and it is not known if there are plans to create a similar R\&D support program or not. US PVC industry is also promoting recycling [10] but does not have a similar R\&D support program now. In the developing world, many PVC products are recycled on commercial basis. A study in Thailand found that not much PVC products were found in the waste disposal sites [11].

Japan's R\&D support program was founded in 2007. Since then, seven projects have been completed and three of them are currently in operation. They are either open new ways to materials which are thought difficult to recycle or develop new applications for recyclates.

\section{Specific PVC recycling technologies developed under the $R \& D$ program}

(a) Separation of PVC compounds from fibers

(Figs. 1, 2)

The project was adopted in 2008 and successfully completed in 2010. It was a unique technology proposal to separate PVC compounds and fibers as they are found in products such as tarpaulins and wall coverings by hitting chopped pieces of composite material with fins attached to a high speed rotating drum. The fins hit the chopped pieces at a speed of some $200 \mathrm{~km} / \mathrm{h}$. PVC compounds and fibers are detached and separated from each other through their different specific gravities [12].

It was an innovative recycling technology proposal for PVC composite products which had been considered difficult to recycle mechanically. The wall coverings industry has long been looking for an effective means to recycle their products-both edge-cuts at production or off-cuts at the construction and refurbishment sites, and post use products-and showed strong interest in the proposal. The proposal was seen potentially promising for the edge-cuts and off-cuts.

The project proposer was a small venture which had been developing various types of machines to sell.

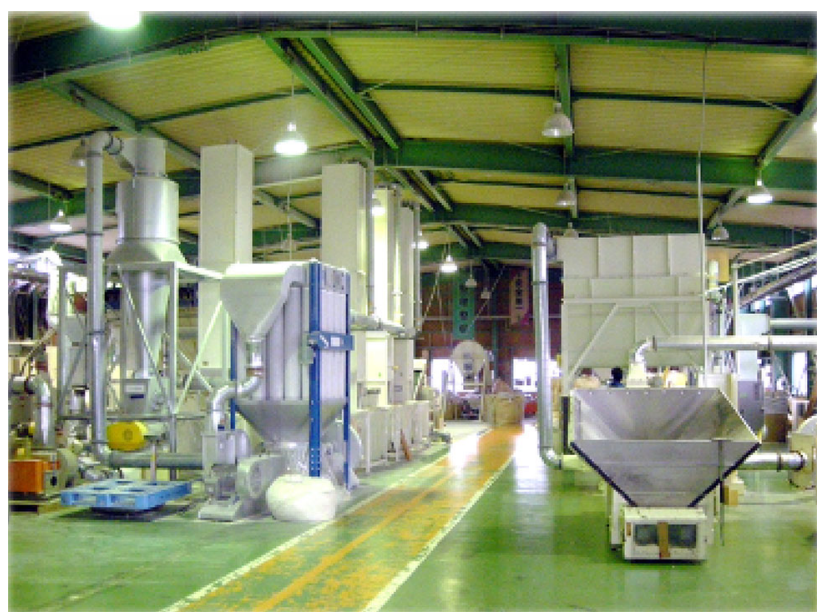

Fig. 2 Photograph of the material recycling facility for wall coverings in operation (Shinwa Kankyo, Co. Ltd. Chiba Recycle Center)
Fig. 1 Diagram of the machine used for the separation of PVC compounds from fibers

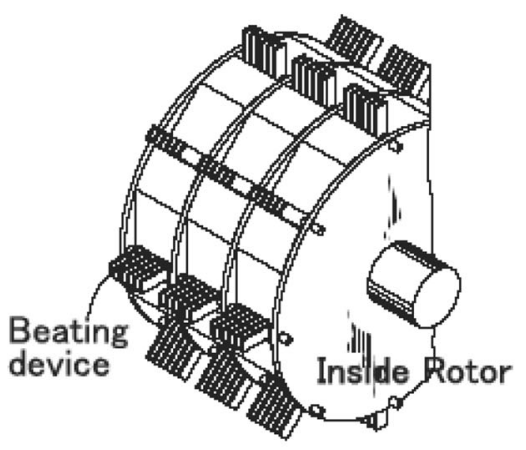


Searching for best conditions and testing durability and safety of operations were major elements of the R\&D proposal. As the technology was so unique and interesting, the project proposer received funding from the local government.

The technology was successful and proved promising. The separation worked beautifully as separated PVC compounds contained only $1 \%$ of fibers, and the fibers contained only $1 \%$ of PVC compounds when thoroughly separated. However, it took time to find out how the technology could be economically adopted in an actual business scenario. Finding stable supply sources of wall coverings or tarpaulins as well as the users of both the separated PVC compounds and fibers was necessary. The original hope of selling machines was an enormous challenge. In the meantime, the machine manufacturer got involved in a chain bankruptcy.

Fortunately, the technology attracted the attention of a waste processing company (Shinwa Kankyo Co. Ltd.) which had a clearer idea than the machine maker of how wall coverings or tarpaulins could be collected and the separated compounds and fibers could be used. The company made efforts to improve the machine, particularly its durability, as well as the material feeder in order to efficiently chop the wall coverings. It plans to additionally treat tarpaulins in the future. It started commercial operation in September 2011 and runs several machines in parallel with a capacity of processing 300 tons/month of wall coverings [13]. The technology was later proved to be effective to process used wall coverings and the company is now trying to expand their sources of raw materials.

The separated PVC compounds have various applications such as floorings, tile carpets and mats. The fibers are short in lengths and not good enough to make fabricated goods or non-woven papers. They were first used as material for cat litter. Later, in collaboration with a PVC product manufacturer the company developed lightweight coasters and bath mats with the PVC compounds and fibers plus virgin resin materials. The compounds received the "Biomass Mark [14]" as they contain recovered biomass in the form of fibers. Then, it also worked with another PVC product manufacturer (Link Planet Co. Ltd.) and developed thick curing sheets made of the separated PVC compounds (30\%), and cable sheaths (70\%) $[15,16]$. The sheets are lightweight compared to metal sheets and therefore easy to move. Nevertheless, they can endure the weight of heavy machines. They are more flexible and resilient and thus do not break easily, and more fire-retardant compared to similar products made of polyethylene [17, 18]. They are now being used at construction or temporary event sites.

The separation machine still needs improvement, in particular regarding durability. Since the rotor spins very fast, the axis may experience metal fatigue. Improvements

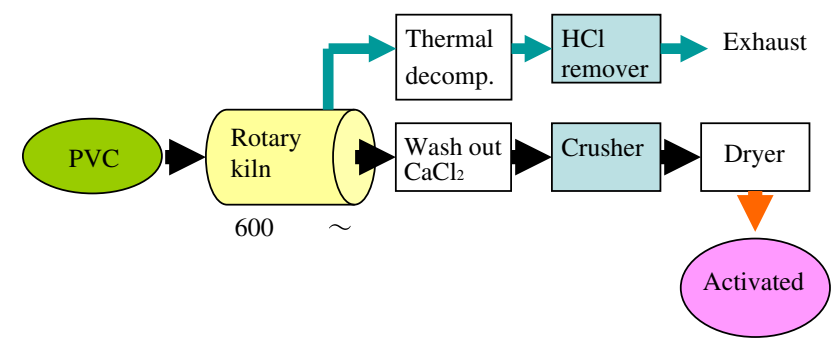

Fig. 3 Flow chart of the production of activated charcoal from wall coverings (Kureha Ecology Management Co. Ltd.)

in machine design or choice of materials for the machines and further adjustment of the operation conditions may be needed. In addition, effective removal of cutter blades which are often disposed together with used wall coverings remains an issue to be addressed.

(b) Production of activated carbon from wall coverings (Figs. 3, 4)

The proposal was to process wall coverings in a rotary kiln to collect hydrochloric acid and activated carbon. It received high expectation by the wall covering industry as it was expected to provide a solution to recycle post-use wall coverings, almost all of which were considered to be sent to landfill. It was adopted in 2008 and completed in 2009.

A typical wall covering is composed of some $30 \%$ of PVC resin, $20 \%$ of additives including plasticizers, stabilizers and pigments, $25 \%$ of filler (calcium carbonate) and $20 \%$ of paper. PVC resin, plasticizer and paper are organic substances and thus were attempted to be recovered as organic residuals after pyrolysis. The calcium carbonate was expected to capture much of the hydrogen chloride and to enable reducing the use of alkali agents to neutralize the products.

Objectives of the R\&D included determining the best operating conditions, such as heating temperature and volume of air inflow, which would allow obtaining the targeted yield of $75 \%$ of thermal energy recovery as organic substance. Other set objectives included verifying the quality of the products and to ensure that the process would not generate harmful substances. In particular, the air flow and heat control were essential to obtain quality products in volume. The wall coverings were heated at a temperature of $600{ }^{\circ} \mathrm{C}$ for approximately $30 \mathrm{~min}$ under a very low oxygen concentration ( $0.2 \%$ or less) as suggested from previous research $[19,20]$.

The technology was proved effective and the resulting charcoal showed good properties as an absorber, in particular in the removal of dioxins from water, and was patented [21]. The yield of charcoal in the trials, however, 
Fig. 4 a Wall covering chips fed into the process, and $\mathbf{b}$ the resulting products consisting of activated charcoal
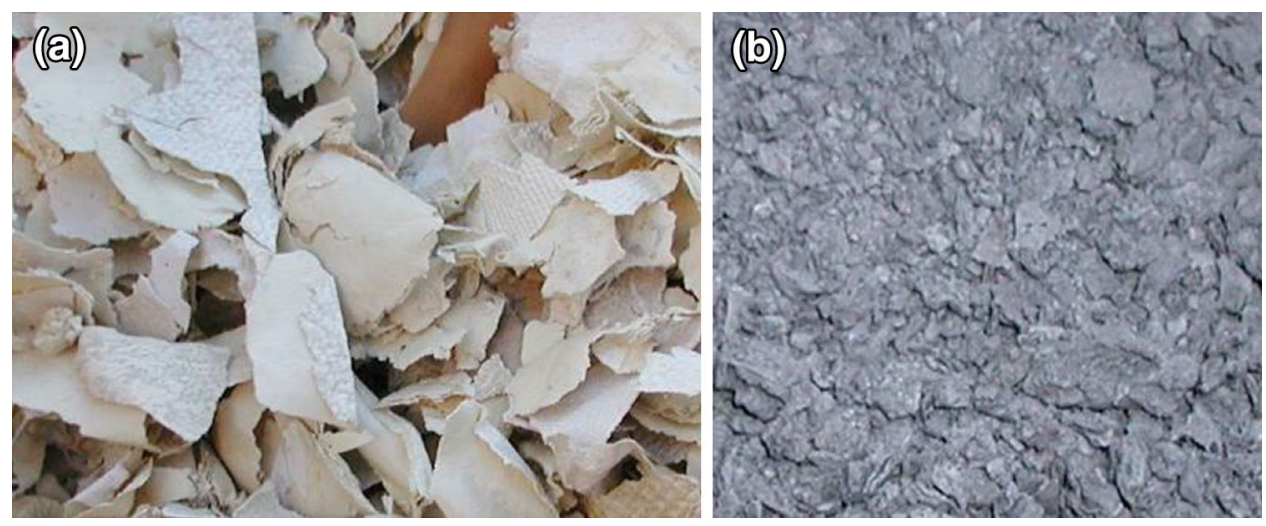
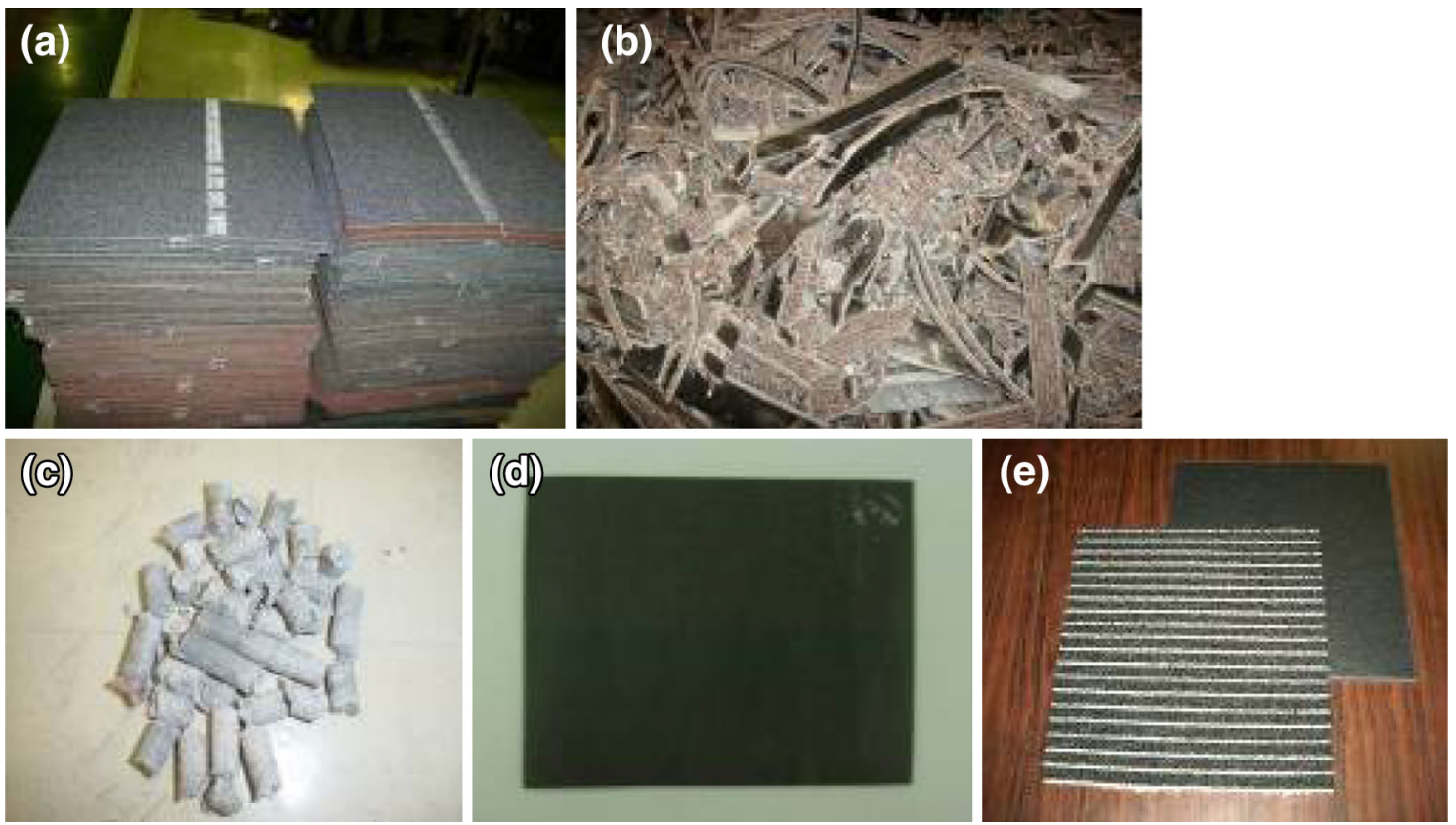

Fig. 5 Stages in the recycling of carpet tiles; $\mathbf{a}$ used carpet tiles, $\mathbf{b}$ edge-cuts of carpet tiles, $\mathbf{c}$ pellets made of edge-cuts, $\mathbf{d}$ layer sheet made from the pellets, and e new carpet tiles using the layer sheet

did not meet the target and remained around $1 / 3$ of the original organic content. The lower than anticipated yield was due to the difficulty in controlling air flow to limit supply of oxygen. The existence of cutter blades and plasters in the used wall coverings caused a problem. The technology has not yet moved on to commercial application due to the reasons discussed later in this paper.

The system needs to be, and it can be, improved to increase the productivity, though additional experiments are necessary. The economy of operation is very much dependent on the waste-processing fee the company can charge and the price of the products. It is considered that there exists a potential; however, the current market situation is not much favorable for the operation of this process. (c) Mechanical recycling of tile carpets by producing pellets from both fibers and PVC compounds (Fig. 5)

The proposal was to produce pellets made from both fibers and PVC backings of the tile carpets [22]. It was adopted in 2008 and successfully completed in 2009.

Tile carpets are a major product as application for recycled PVC compounds. Used PVC accounts for 60-80\% [23] of the PVC compounds used in the tiles, or more than $43 \%$ [24] of the total weight of the tiles. Carpet tiles made from significant amount of recyclates are qualified as eco-mark products. There already is a commercially operated technology which peels off the backing of tile carpets to collect PVC compounds with a capacity of 18,000 tons of carpets/year [25]. Through the use of this 

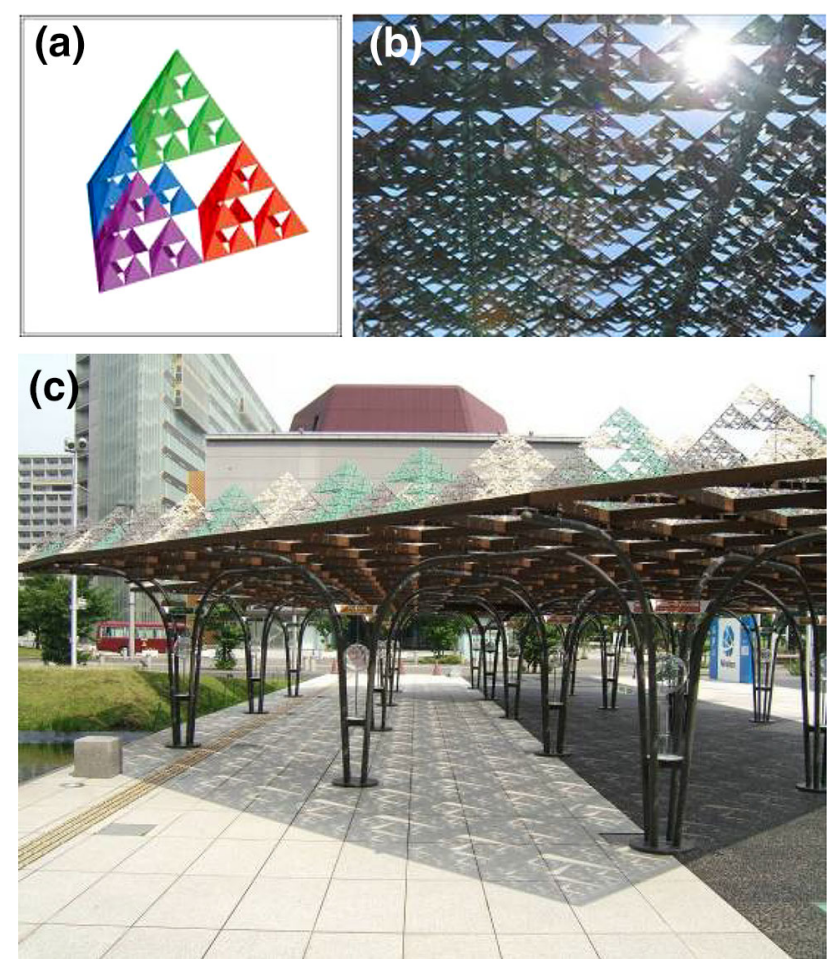

Fig. 6 Photographs of PVC shades; a modular unit shape, b shades composed of various modular units, and $\mathbf{c}$ installation of the shades and their resulting shadows

technology PVC compounds can be recycled a number of times as backing of tile carpets.

However, it was difficult to find applications for the fibers removed from the tile carpets. The project proposal was to produce pellets, using both fibers and PVC backings, which could be used as a layer for the baking of carpet tiles. A major objective was to reduce the amount of wastes disposed at the production sites of carpet tiles. The amount of edge-cuts can be as much as $5 \%$ of the total volume of production at the production sites. In addition sub-standard products are generated, which add up to the portion that can be recycled.

The goal of the R\&D was to ensure the quality of the pellets with continuous operation. Adjustment of the conditions of kneading and additives were major elements for test operations. The technology was proved successful, transferred to a waste service company which now processing around 100 tons of post use products per month.

(d) Air-permeable shape shades made of used PVC (Figs. 6, 7)

The proposal was adopted in 2009 and the R\&D was completed in 2011 [26]. The proposal was to create a completely new product which had not existed in the past:

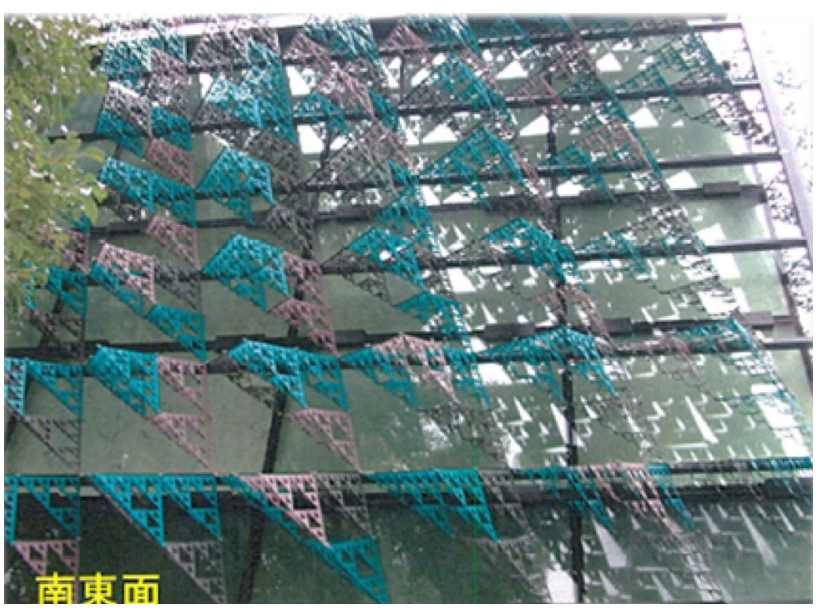

Fig. 7 Performance test of PVC shades used as blinds for windows

air-permeable shape shades made of used rigid PVC. The properties of rigid PVC, in particular its durability and its relative easiness to form complex structures, were made good use of. The shades consist of small triangular-shaped pieces which imitate tree leaves. The leaves are small enough to be cooled by air currents or wind which passes through them. The heat absorbed by the leaves is removed by the air currents which convect upward, in contrast to flat structures like tents or roofs which emit the absorbed heat from sunrays to their surroundings. The idea had been developed by Satoshi Sasaki, professor at Kyoto University [27] and patented [28]. The project proposer had a patent licensing agreement with the professor.

The shades have multifaceted environmental properties. First, they can contribute to abate heat island effects by suppressing buildings to accumulate heat and lowering the surrounding temperature. Second, they do not require water supply, which is necessary for trees. Additionally, they do not require maintenance such as trimming the trees and cleaning of fallen leaves. Finally, the application opens a way for recyclates to be used for highly value added products which were otherwise used for down-graded products. And the use of recyclates increases the environmental value of the product.

Major objective of the R\&D was to test the quality as well as the productivity of the products with increased amount of PVC off-cuts and to confirm the performance of the products as shades.

Single triangular-shaped units were produced by injection. Four single units were then assembled to make a modular cluster. Clusters were placed horizontally in a position such as to shade most of the sunrays at noon.

It was confirmed that as much as $70 \%$ of PVC off-cuts, mostly from PVC pipes and window frames, together with virgin PVC resins could be used for injection. 
The shades showed a remarkable performance. The ground temperature under the shades was reduced by $10{ }^{\circ} \mathrm{C}$ while the surface temperature of the shades did not considerably rise above ambient temperature. The structure was not damaged by the strong rains and winds of a typhoon. The air-permeable shape appeared to help avoid direct hits from the wind [29].

Major challenges were (a) securing stable supply sources of quality PVC off-cuts, (b) increasing the productivity, and (c) ensuring durability.

With regard to productivity, new molds were developed later to form a modular cluster of leaves with a single injection. However, the portion of off-cuts in the product was reduced to some $30 \%$ to ensure quality of the products due to the constraint of the supply of quality off-cuts. The biggest difficulty was the supply of quality recyclates to ensure productivity and the quality of the shades. In Japan, most of the waste at production sites of pipes and windows are recycled within the same facility and not many of them are traded. There were limited amount of recyclates which could be purchased in the market, and it was difficult to know exactly what additives and how much of them were used. Stains appeared on the surface of the products, which were found to be caused by the mixing of recyclates with led stabilizers and those with tin stabilizers. When imported pellets of used PVC were used, the shade structure was distorted by the heat of the sunrays. The problem was caused by a slight amount of plasticizers which were found to be included in the pellets. The first shades tested were found to be brittle after a couple of years of exposure, implying that both the thickness of the pieces and the shapes of the joints should be improved.

Currently, durability tests continue and products using PVC off-cuts have not been commercially manufactured yet. In parallel, trials have been conducted to test the effectiveness of the products by placing them on the westfacing building walls at the Osaka City University, to shade them form the afternoon sunrays.

(e) Reuse of PVC backings from tile carpets as extender resin (Fig. 8)

The proposal was adopted in 2009 and completed in 2010 [30]. The proposal was to re-compound removed backings from tile carpets to expand the possible applications such as extender resin for mats or other appropriate uses, and by thus, to reduce the use of virgin materials. The PVC compounds were to be separated from fibers mechanically by beating chopped waste pieces of tile carpets. In the test runs, PVC compounds scratched off from tile carpets were used. In the laboratory testing, PVC compounds could be mixed well with additives to adjust viscosity and other properties. The expectation was that the re-compounded
PVC could be used for various types of applications by adjusting the amount of additives and the conditions of recompounding.

The idea, however, was found much more difficult than thought at the outset. Part of the recovered PVC compounds degraded by heat generated in the mixing process, and thus generated bad odors. Furthermore, the amount of additives needed to adjust the properties of the processed compound was more than anticipated. Because of these problems, the cost was found to be higher and the quality was poorer than anticipated. Unfortunately, it was not possible to find a desirable application of the separated fibers.

(f) Dechlorination of automobile shredder dusts residues (ASR) to make charcoal for cement production (Fig. 9)

The proposal was adopted in 2011 and completed in 2012 [31]. Its purpose was to dechlorinate automobile shredder dust with ordinary pressure steam and alkali (slaked lime or sodium bicarbonate) in an antler kiln and collect organic residuals which can be used to produce cements. The main objectives of the $R \& D$ were to find most appropriate condition and to check that waste water after treatment of ASR would meet the environmental standards, in particular, those regarding residual oily matters. The targets were to reduce chlorine content to less than $1 / 5$ of the original value at a cost less than $10 \mathrm{JPY}$ (US 10 cents) $/ \mathrm{kg}$ while retaining an organic content as feedstock more than $70 \%$ of the original ASR. The test trials proved that all these targets were met.

The amount of ASR generated was estimated to be 550-750 kton in 2005 when End-of-Life Vehicle (ELV) Recycling Law was enacted [32]. The law aims to recycle as much as $95 \%$ of the materials of a disposed car by 2015. To meet the overall target, $70 \%$ of ASR should be recycled by then. ASR were treated to recover energy in the form of heat or organic gas and to collect metal materials [33]. ASR basically consists of plastics of various parts made of various plastics. The ASR usually includes some $2 \%$ of chlorine. Suppose all chlorinated substances are associated with PVC products, this suggests that the PVC content in ASR is somewhere around $4 \%$. ASR is expected to be used as material for cement production if chlorine content is reduced with reasonable cost. According to the plan of the proposer, some 20,000 tons of ASR could be processed a year for cement production.

There are several ways to remove and/or recover chlorine from plastics wastes. Gasification at a temperature around $600-800{ }^{\circ} \mathrm{C}$ is one way to do so but a challenge is the troubles caused by pyrolysis tar [34]. Heating PVC only does not produce much tarry matters. However, when other 
Fig. 8 Schematic chart of the reuse of PVC backings

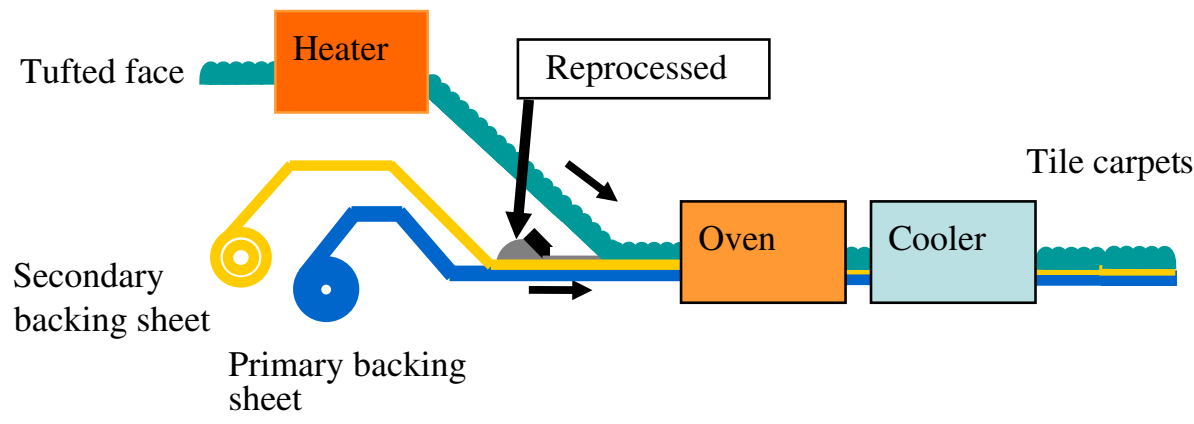

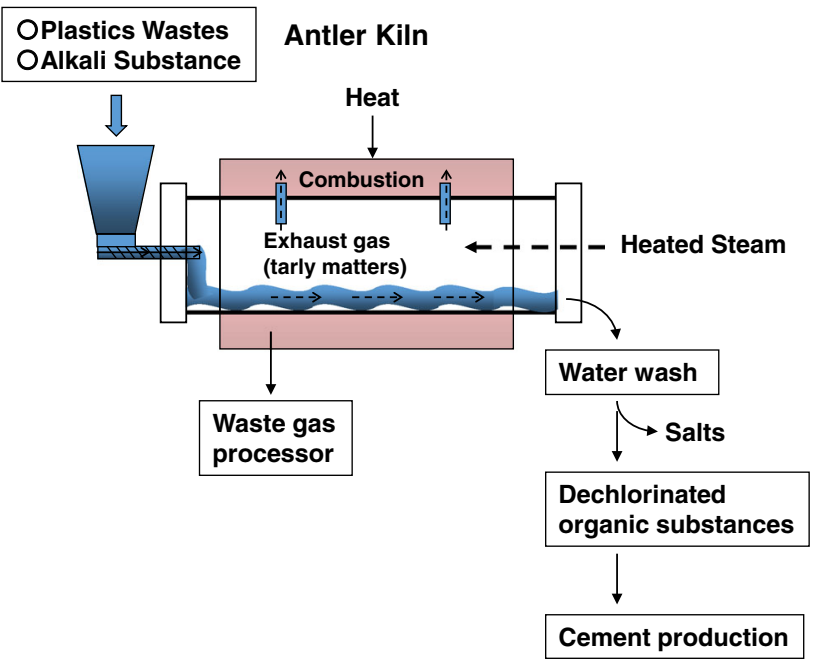

Fig. 9 Schematic chart of the dechlorination of ASD

plastics are heated together, tarry matters are generated and make it difficult to collect easy-to-use dry organic matters for cement production. As chlorine content of the fed plastics increases, it may become possible to separate chlorines as hydrogen chloride by decomposing the plastics with heat at around $250-300{ }^{\circ} \mathrm{C}$ [35], avoiding higher temperatures that might cause the breakdown of the polyene structure of the dechlorinated PVC [36]. When steam is used as a medium for the reaction, hydrogen chloride can be recovered as aqueous hydrochloric acid. An innovative alternative process that has been investigated involves wetprocessing of the ASR containing PVC using an ethylene glycol solution as the reaction medium in a ball mill [37]. In this process, an improved efficiency was obtained through the high boiling point of ethylene glycol (around $196{ }^{\circ} \mathrm{C}$ ), which allows the dechlorination process in-solution to operate at a moderately high temperature at which the polymer matrix is not affected by thermal decomposition. When a medium such as ethylene glycol is employed, the chlorine can be retrieved through methods like electrodialysis with ion-exchange membranes [37], or the use of alumina/zeolite membranes [38, 39].
Either of these approaches was not taken by the proposer as it could not find appropriate use for hydrogen chloride in their factory or vicinity. The reason why an antler kiln was chosen was it could easily remove tarry matters by evaporation. In addition, the process has an advantage to be able to use ordinary pressure steam (at around $240{ }^{\circ} \mathrm{C}$ ), which may enable them to take advantage of heat generated in the cement manufacturing process. The chlorines were trapped by the alkali and washed away from the process. Additionally, the addition of alkali can help reduce the amount of problematic tar, and it has also been reported that the presence of alkali such as $\mathrm{NaOH}, \mathrm{Ca}(\mathrm{OH})_{2}$ or $\mathrm{Na}_{2} \mathrm{CO}_{3}$ accelerate the gasification reaction of $\mathrm{PVC}$ and dehydrochlorinated PVC as well as increase the yield of desirable gas products such as hydrogen [40].

The economic potential of the technology is being studied. It was found that several times more alkali than the equivalent amount was needed to reduce the chlorine content sufficiently. However, since the added alkali becomes part of the cement materials the costs were not very sensitive to the amount of alkali substances fed into the process. The fee for processing ASR ranges from 20 to $30 \mathrm{JPY} / \mathrm{kg}$ in Japan. The process costs with the above technology depend on volumes and continuity of supplies. Transportation costs of ASR also need to be considered.

\section{(g) Separation of PVC films from fibers (Fig. 10)}

The proposal was adopted in 2011 and completed in 2012 [41] and consisted in peeling off PVC films out of sheet edge off-cuts of tarpaulins, vinyl leathers, and vinyl curtains. In the production facilities, edge off-cuts amount to $5-10 \%$ of the products. In addition, certain amounts of substandard products and unshipped products occur in the factory. These contain quality PVC compounds but there was no adequate way to separate and use them as materials. Vast majority of off-cuts had been sent to landfill and not recycled.

The products have two layers-a vinyl film and a textile sheet, or else three layers-vinyl films used for both surfaces and a textile sheet in between as enforcer. In many 
Fig. 10 Photograph of the separation machine for PVC tarpaulin cut tapes. a Overall view of the equipment; the edge of the cut tape (left) is heated between the slits before PVC and textile layers are separated (right). b Equipment in operation; Edge cut tape (right) slit heater (middle), and peeled off PVC (left). c Feed material and obtained products; separated PVC (top), separated textile (middle), and original edge cut before separation (bottom)
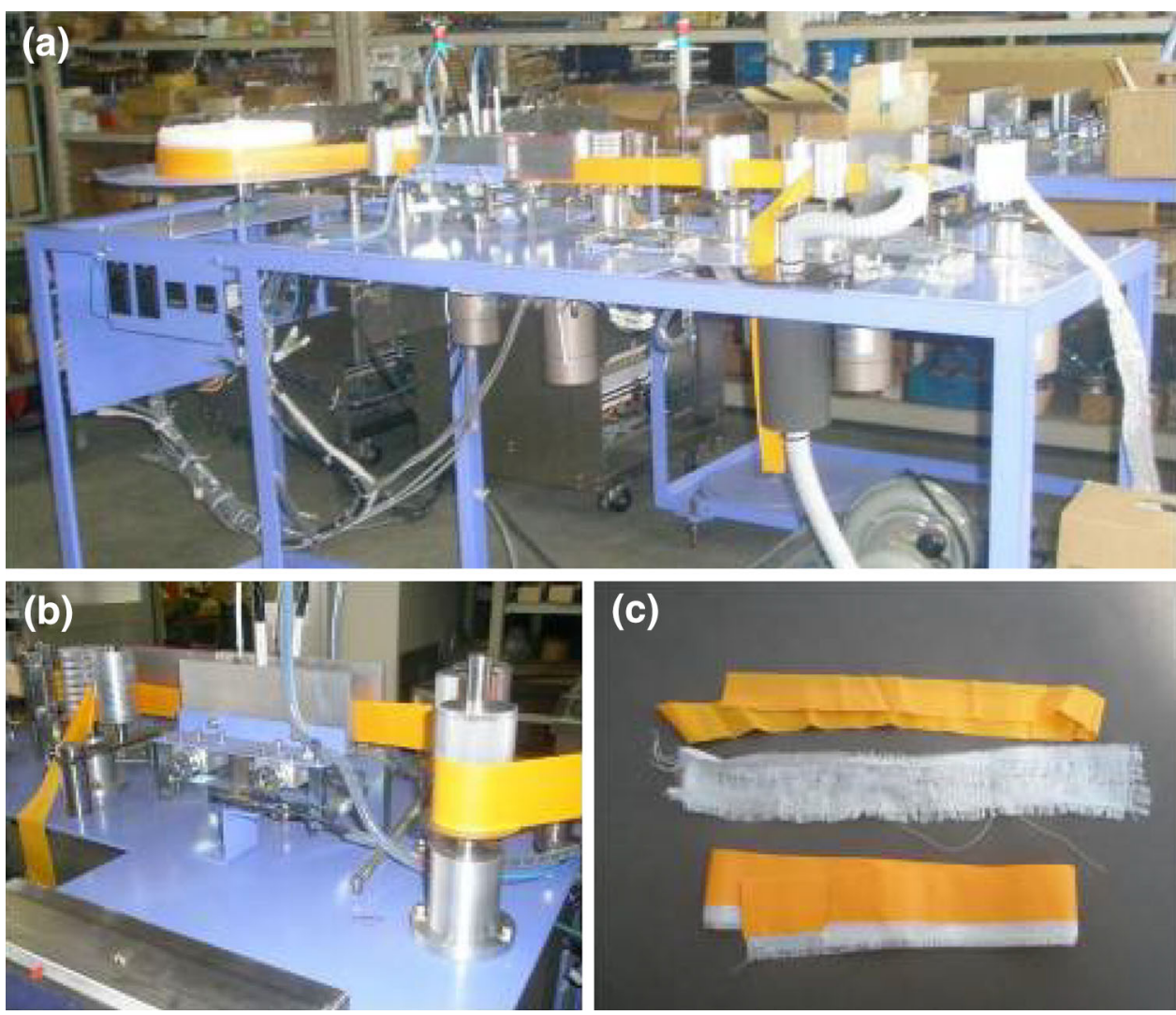

cases, the PVC sheets are crimped into the textile sheets, therefore, they can be peeled off when heated, given that they are attached without adhesives.

The edge off-cuts are wound at the sheet production site into belt-shaped rolls. One of the edges of the off-cut rolls was pulled and heated between slits in order to weaken the bond between the PVC sheets and textiles. The heat temperature depends on the material and the room temperature. It ranges from 40 to more than $100{ }^{\circ} \mathrm{C}$. Afterwards, the PVC layer and textile layer are peeled off and rewound. The speed and tension to feed and pull the tape also depend on the material of the tape. The objective of the R\&D was to find the best conditions of heating, adjusting pulling tension and speed of processing.

The technology was proved effective and patent applications were filed. The economics of the technology is under scrutiny and the high value applications of the products need to be searched. It is considered essential to raise the productivity, and an effective method needs to be developed so that the edge off-cuts can be automatically fed to the process. Wide pieces need to be sliced so that they can be rolled into rolls of less than some $20 \mathrm{~cm}$ width. PVC compounds peeled off from tarpaulin, curtains, or vinyl leathers are of high quality and some of them may be used to produce similar or slightly lower grade products. Although the peeled PVC is clean, the compositions of additives vary among products, making it difficult to find higher value applications other than flooring (plasticized PVC).

\section{Conditions for success}

Successful projects enjoy favorable conditions besides technological success. First, it is always important to find stable supply source(s) of quality materials such as offcuts, wasted or used products. Second, there must be users who purchase the processed materials or products. Third, the processed materials or products need to be cost-competitive. The cost-competitiveness depends on various factors including the price of the source materials, costs of transportation, storage or other logistical arrangements for material supply, as well as product delivery.

These three factors are usually interrelated. When supply sources are clustered in a limited area, transportation costs of the materials can be reduced. If such supply sources constantly or regularly generate wastes, off-cuts, or used products, economic transportation can be easily arranged. Supply stability of the raw materials helps increase the productivity and reliability of the quality and delivery of processed materials or products. An extremely well suited condition is that supply sources take back the 
processed materials or products. If the total cost of recycling is more than the profits, or if the amount of money saved as compared to the existing practices, such as for example sending to landfill, then the technology will not be commercially used. Slight amount of premium costs may be acceptable since recycling may earn good social images. The costs for landfill vary among regions in the country and are in a range between 10 and 20 yen (US cent) $/ \mathrm{kg}$ and that for incineration is between 30 and $40 \mathrm{yen} / \mathrm{kg}$. Both do not include costs for transportation and can fluctuate by economic situations or other reasons.

\section{(a) Supply of off-cuts/wastes/post use products}

Off-cuts or wastes generated in production facilities are usually good sources of supply for recycling in terms of quality. However, due to this very reason, much of such products are recycled within the factory. Nowadays, companies are trying to dispose of waste as little as possible. Many companies try to achieve a so called "zero-emission". Therefore, the amount of off-cuts or wastes generated in the production facilities and which circulate in the recycle market appears decreasing according to the hearings from the waste service companies. Vinyl sheets for agricultural use are an example. Reduction of the volumes of wastes emissions has become more challenging to existing recyclers these days. On the other hand, for some product groups, there are significant amounts of off-cuts or wastes which are sent to landfills due to the absence of adequate recycling technologies. Wall coverings, tarpaulins, vinyl leathers are among such examples. The volume of wastes generated at the production sites including offcuts and sub-standard products is estimated at as much as $7 \%$ of the products. Some $15 \%$ of the products shipped are estimated to become waste as off-cuts at the construction sites. In addition, used wall coverings are emitted on the spot.

Wall coverings were considered difficult to recycle in the past. Share of vinyl products accounts for more than $90 \%$ of the market. Some 110,000 tons of waste is estimated to be emitted of which 100,000 tons is generated at the construction sites and the rest at the production facilities [42]. The Japanese Wall Coverings Association has long been searching for adequate recycling technologies for used products. It is because wall paperhangers are usually asked to bring back used wall coverings removed on the spot. However, a legal license as a waste collector is required to accept wastes for disposal. The Ministry of Environment issues special permits to those who manufacture or sell products to collect the used products if they are better suited to treat the products due to their unique capability or better knowledge of the products than municipal garbage collectors. Those who receive the permits can move the collected used products beyond the borders of prefectures to process the collected products more efficiently at the most suited facilities. The association appealed that it could do better than others in recycling by the separation technology shown in (a) above and is trying to obtain the permit by 2014 .

The situation that around $60 \%$ of the vinyl wall covering production is concentrated in the Kanto area of Japan is a favorable condition to establish a system in the area for collecting waste wall coverings generated at factories. However, an effective collection system needs to be developed to collect off-cuts and used paper emitted at the construction sites.

Significant amounts of wastes are generated at the production sites of tile carpets as well as at the construction sites. In contrast to wall coverings, used tile carpets can be easily removed and collected for recycling. The separated PVC compounds can be used as material for tile carpet backings or mats. Therefore, there are already commercially operated recycling facilities for used tile carpets. Since the sizes of the tile carpets are standardized, processing the used products is mechanically easier than the edge-cuts and wastes at production sites. However, it is difficult to find appropriate applications for the removed fibers. This was a major reason why project (f) was proposed.

Supply of quality off-cuts or wastes was a bottleneck for project (d), involving PVC shades. Used products were not considered suitable for shades due to necessity of quality assurance. Therefore, quality off-cuts and wastes of pipes and window frames generated at the production sites were searched for. However, most of off-cuts and wastes of these products have already been recycled in the factory and it was difficult find stable supply sources in sufficient mass. This was a major reason why imported pellets of used PVC were tried, but were found inadequate due to the existence of plasticizers as described earlier in this paper.

Quality control is a challenge in some cases due to the fact that a variety of additives have been used in PVC products in the past and it is not easy to identify what they are, in particular for products which were used for decades. The quality of products may be compromised if used PVC products with different additives are mixed. For example, stains appeared on the surface when PVC products with tin stabilizers and those with lead stabilizers were mixed to make test pieces of PVC shades.

(b) Existence of users who purchase the processed recyclates

Several conditions need to be met to find users who purchase the recyclates. First, the properties of the products need to satisfy the needs of the users. Second, supply 
should be stable in terms of quality and quantity. Third, the price of the recyclates should be sufficiently high and enough to be able to cover the processing costs. The costs are influenced by the transportation conditions which are discussed in the following section.

When recyclers are qualified as waste service companies, they can collect the used products asking for fees to process them. The waste service companies can reduce the price of the processed products by charging processing fees. According to the Waste Disposal and Public Cleaning Law in Japan, if one is not qualified as a waste service company, then one cannot charge fees for waste treatment. To accept the used materials, it is needed to "purchase" the used products as "materials" and thus the price of the processed products increases.

When the processed products are taken back by the partner who generates the products, recycling may work as long as the costs for processing are at an acceptable level for the emitters. However, if the users are different from the emitters as is the situation in most cases, high price of the processed products becomes a barrier to recycling.

This is the case of the inventor of the technology of the project (a). The inventor was a machine manufacturer who wanted to sell his machines and did not have the license as a waste service company. Therefore, he needed to purchase wall coverings waste to test the machines. Unfortunately, the company could not find those who would purchase the separated PVC compounds due to their high prices. Later, the technology was purchased by a waste service company which charged processing fees for the wastes and could reduce the price of the separated PVC compounds. It took advantage of its experiences and network to find users of the PVC compounds as well as the separated fibers.

Several different users may need to be found for effective recycling. Applications can vary depending on the quality and specifications of the processed recyclates. In the case of pipes and fittings, high quality post-use products reborn as new pipes and those of lower grade are exported to produce pipes, mats or the soles of shoes at the destinations. For PVC textile/fiber composite products like wall coverings and tarpaulins, applications need to be found for separated PVC compounds and textile/fiber, too. On average, vinyl wall coverings contain some $30 \%$ of vinyl resin, $15 \%$ of plasticizers and $26 \%$ of fillers. All of these together as a compound can be used as part of vinyl floorings, for example. The wall coverings also contain some $21 \%$ of paper pulp which is bulky. It is usually difficult to find applications of textiles/fibers because the fibers are too short. Recovered fibers may be kneaded into mats or used as material for cat litter. Since the value of fibers for such use is very low, economic recycling can be possible if PVC compounds are priced high enough. On the other hand, if long fibers can be recovered, such fibers may be used for applications such as cushioning and packaging materials. Now, there is a trial of new technology which does not chop fibers too short. This may become a promising new project in the future if the adequate applications for the fibers can be found and the fibers can be sold at high price.

\section{(c) Collection, transportation, and storage costs}

Transportation and sometimes storage costs can become significant barriers to recycling. The costs are affected by the locations of emission points, the distance between emission points and the processing facilities, and the regularity of waste availability. In the past, the PVC Pipe and Fitting Association which has a voluntary recycling scheme, used storage points for collected products. However, the operational and storage costs of the system were significant. Therefore, the association decided that it would stop using intermediate storages and send collected products directly to the waste service companies now.

In case of wall coverings, waste arising occurs at the production and construction/refurbishment sites. The wastes or off-cuts at the production sites can be easily collected. However, transportation costs depend on the distance between the production sites and the waste service facilities. The distance was a cause of higher costs in the specific case of project (b) as the facility is located in Fukushima prefecture, some $200 \mathrm{~km}$ from the center of the metropolitan area. As stated above, the vast majority of wall-covering manufacturers are concentrated in the Kanto area and the facility (b) was located at the edge of the area. However, the products (charcoal) after the processing could not be priced high enough to ensure economic operation.

The machines in the project (a) were originally developed with an expectation of being introduced to wallcovering manufacturers. At present the machines need further improvement for reliability for the purpose. Now, several machines are operated in parallel at one site to be prepared for a sudden need of maintenance. When the reliability of the machine is improved and the price of the machine can be set reasonably, it can be deployed at the production sites of wall coverings.

The situation regarding post-use wall covering is very different. Used coverings arise numerous construction or refurbishment sites but the volume of the wastes arising at a site is usually limited. Therefore, efficient collection and transportation are key conditions for successful recycling. The Wall Covering Association already started preparation to apply to the Ministry of Environment for a permit to operate as waste service provider for used wall coverings and has started establishing a collection system with the technology (a) as a core recycling tool. Without a permit, one cannot receive wastes and move them beyond 
prefectural borders. For the time being, it is planned that used or unused wall coverings will be collected in the Kanto Area or vicinities of the facility of the company in collaboration with the Wall Covering Association. Once the effectiveness of the collection system as well as stable operation of the facility (or the separation machines) are confirmed additional collection networks will be established in the rest of the country with new facilities to cover the new areas.

The economy of a recycling system is influenced also by the level of fees that the wastes emitters are prepared to pay, stability and volume of waste supplies, modes of transportation, as well as the costs of alternatives such as landfill.

\section{(d) Connecting the links}

Usually, it is unlikely that a recycling project proposer has a solid idea of how the materials can be obtained and who will use the processed products. Successful recycling often requires case-specific solutions considering various factors, such as quality and quantity of the wastes as recyclates, processed products, costs of processing and transportation, geographical locations of the emission points, processing for recycling and the users.

The Vinyl Environmental Council (VEC) has a desk for recycling and receives around 30-40 inquiries every year. It has information on hundreds of companies producing vinyl products or providing waste-processing services. It tries to find potential partners for those who want to explore opportunities.

An example of this was the case of material recycling of wall covering described above. When the recycle service company came to VEC for advice on new application, VEC introduced a PVC product manufacturer which was producing cure sheets with post-use sheaths of cables. The cure sheets manufacturer worked together with the recycle service company to develop new cure sheets using the recovered PVC compounds and fibers together with sheaths for construction sites. The new sheets are lighter in weight and easier to handle at the construction or event sites (Fig. 11).

\section{(e) Selection of R\&D proposals}

Selection of R\&D proposals needs to consider not only newness of technologies but also their economic potential, which is influenced by numerous factors discussed above. Indeed, the $\mathrm{R} \& \mathrm{D}$ promotion program is designed to assess proposals considering various aspects. To do so, a committee was established with academic and industry experts as its members [43]. It assesses the innovativeness of the technology, technical feasibility and economic potential of
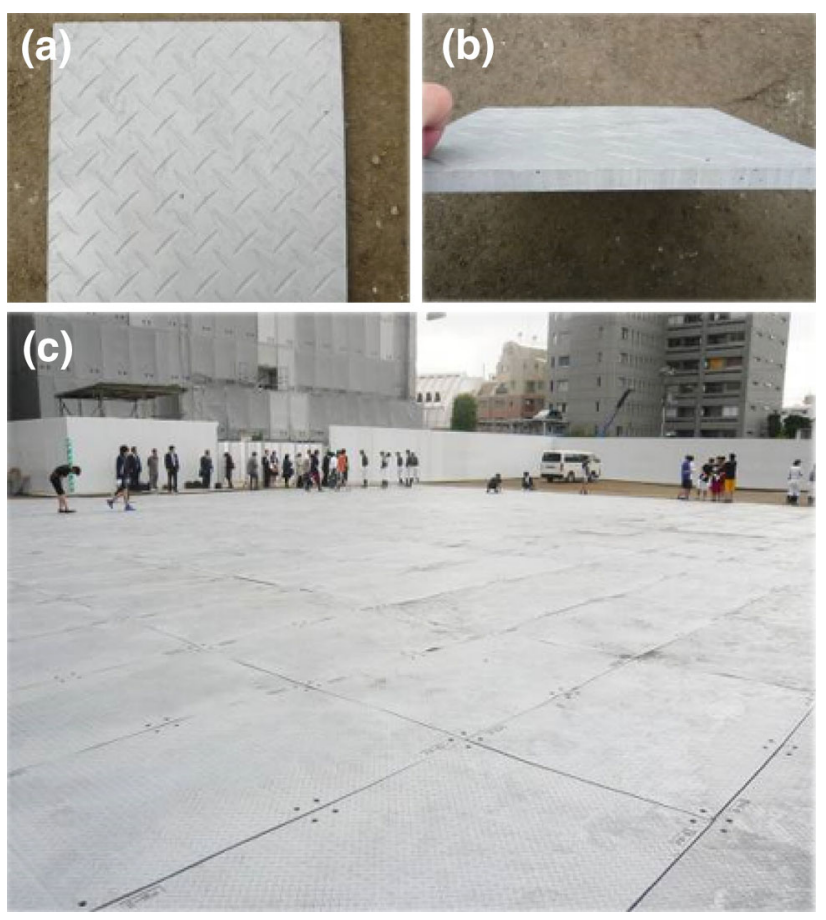

Fig. 11 Cure sheets made of PVC sheaths and recovered PVC compounds from wall coverings; a overhead view of design, $\mathbf{b}$ side view of thickness of the sheaths, and $\mathbf{c}$ vinyl cure sheets made from recovered resin and fibers of wall coverings together with used sheaths of cables in use at site

the proposal as well as the capability of the project proposer. Contribution to improvement of the image of the PVC products may be taken into consideration. The Vinyl Environmental Council makes the final decision whether it adopts a proposal or not based on the assessment made by the committee. In case a proposal is judged as not matured enough, VEC may help the proposer to collect additional data, to conduct further testing, and to prepare feasibility study or find potential partners which supply materials or use the processed products.

It is difficult to prepare quantitative criteria for selection of project proposals as there are so many case specificities. However, there is a general feeling among the expert members of the Assessment Committee for the R\&D and VEC members that the lessons learned though the course of adopting and following the specific projects have contributed to the assessments of new project proposals.

\section{Conclusion}

The R\&D support program in Japan to promote recycling of PVC materials is a unique industry-led initiative and has shown significant outcomes in developing new technologies and applications; three out of seven projects have 
become commercially operative, and a couple of them have proved technically feasible with economic potential. There is room for improvement for all of these, suggesting possibilities of wider applications in the future. It is noteworthy that promising solutions were found for difficult-torecycle PVC materials such as wall coverings and tarpaulins-a common issue that the rest of the world has shown interest in.

The success of a project proposal is case specific depending on various factors including stability of supply of off-cuts, edge-cuts or post-use materials, the existence of users, the quality and the prices of the processed products, transportation and storage costs. Therefore, not only technical feasibility and economic potentials but also surrounding conditions need to be studied to find out promising project proposals, although it is difficult to set quantitative criteria for the selection. Linking players from upstream to downstream is a key for successful operation and the role of mediator to connect links is often important.

Open Access This article is distributed under the terms of the Creative Commons Attribution License which permits any use, distribution, and reproduction in any medium, provided the original author(s) and the source are credited.

\section{References}

1. PWMI Newsletter (2013) Plastic Waste Management Institute, Japan, Vol 42. http://www.pwmi.or.jp/ei/siryo/ei/ei_pdf/ei42.pdf. Accessed 1 Aug 2013

2. Dioxins (2012) Government of Japan. http://www.env.go.jp/en/ chemi/dioxins/brochure2012.pdf. Accessed 1 Aug 2013

3. Wielgosinski G (2010) The possibilities of reduction of polychlorinated dibenzo-p-dioxins and polychlorinated dibenzofurans emission. Int J Chem Eng. doi:10.1155/2010/392175

4. Buekens A, Cen K (2011) Waste incineration, PVC, and dioxins. J Mater Cycles Waste Manag 13:190-197

5. "Recycle Vision" of the Japanese PVC industry. It was first issued in 2007 and revised in 2012. http://www.vec.gr.jp/lib/pdf/ recycle_vision.pdf. Accessed 1 Aug 2013. PVC Industry Issued the "PVC Recycle Vision", PVC News (2007) vol 62. http://pvc. or.jp/news_ind/62-1.html. Accessed 1 Aug 2013

6. Buekens A, Sevenster A (2010) Vinyl 2010-nearing the target date. J Mater Cycles and Waste Manag 12:184-492

7. Sevenstar A (2012) Vinyl Plus, the new European PVC industry's voluntary programme toward sustainability. J Mater Cycles Waste Manag 14:281-285

8. PVC Recycling Technology R\&D Support Program established by the Vinyl Environmental Council in 2007. http://www.vec.gr. jp/recycle/recycle5_2.html. "R\&D Support Program for PVC Recycling”, PVC News (2008) vol 64. http://www.pvc.or.jp/ news_ind $/ 64-1 / \mathrm{html}$. Accessed 1 Aug 2013

9. "Voluntary Commitment" page 10 (22 June 2011), Vinyl Plus. http://www.vinylplus.eu/uploads/Modules/Publications/vinyl plus_voluntarycommitment_2011.pdf. Accessed 6 Nov 2013

10. Recycling of PVC materials in the United State can be found in the web of the Vinyl Institute. http://vinylinfo.org/recycling/. Accessed 6 Nov 2013
11. Nujarin Ramankul, "Thailand PVC Recycling case study" (9 June 2013), ASEAN Vinyl Council

12. "Material recycling system for waste composite plastics" R-Inversatech Ltd. Tokyo Showcase. http://www.a-concept.co.uk/ tokyoshowcase2009/participants/rinversatech.pdf. Accessed 1 Aug 2013

13. Recycling of Wall Coverings by Shinwa Kankyo Ltd., PVC News (2013) vol 84

14. Those products which contain minimum $10 \%$ dry weight biomass can be qualified to use the "Biamass Mark" that is operated by the Japan Organic Recycling Association. The PVC compound ("Hybrica Biomass Compound") is registered by the Japan Wall Covering Association (No. 100199)

15. Recycling of Wall Covering by Shinwa Kankyo Ltd., PVC News (2013) vol 84

16. http://www.linkplanet.co.jp/product/ps-y.html. Accessed 1 Aug 2013

17. Wiley (2011) Properties and behavior of polymers. In: Ch. Flammability, vol 1. John Wiley \& Sons, NJ

18. Landrock AH (1983) Handbook of plastics flammability and combustion toxicology. Noyes, $\mathrm{NJ}$

19. Kawabata H, Akita S, Ono H, Usui T (2009) Influence of $\mathrm{O}_{2}$ concentration on dioxins formation in combustion processes. J Jpn Soc Exp Mech 9 (Special Issue):141-144

20. Chang MB, Huang TF (2000) The effects of temperature and oxygen content on the PCDD/PCDFs formation in MSW fly ash. Chemosphere 40:159-164

21. Patent 2009-155132, Japan, "Method of manufacturing activated carbonized material and method of treating dioxins"

22. The proposer was Suminoe Textile Co. Ltd. http://suminoe.jp/ english/index.html

23. http://suminoe.jp/interior/products/carpet/ecos.html. Accessed 1 Aug 2013

24. Kawashima Selcon Co. Ltd. The company says that $\mathrm{CO}_{2}$ emissions can be reduced by $18 \%$ by recycling. http://www.kawashimaselkon. co.jp/csr/product.html\#yukaeco. Accessed 1 Aug 2013

25. Recycling of tile carpets by Refine Verse Ltd. PVC News (2006) vol 57

26. The proposer was Sekisui Chemical Co. Ltd. http://www.seki suichemical.com/. Accessed 1 Aug 2013

27. Nakamura Miki, Sakai Satoshi, Onishi Masanori, Furuya Kimie (2011) Thermal radiant environment measurements with fractal blind. J Heat Isl Inst Int 6:8-15

28. Patent 2008-196254 (Japan), Kyoto University, "Sunshade, radiator, and manufacturing method for the same", Patent 2011-021460 (Japan), Sekisui Chem Co LTD, Sekisui Integrated Research Inc., "Molded sunshade member and sunshade"

29. "PVC Shades are tested at the National Museum of Emerging Science and Innovation" PVC News, (2009) vol 70

30. The proposer was Yamamoto Sangyo Co. Ltd. http://www.uniland.or.jp/jci/yamamoto.html. Accessed 1 Aug 2013

31. The proposer was Taiheiyo Cement Corporation. http://www.tai heiyo-cement.co.jp/english/index.html. Accessed 1 Aug 2013

32. Ministry of Economy, Trade and Industry. http://www.meti.go.jp/ committee/downloadfiles/g41109b06_05j.pdf. Accessed 7 Nov 2013

33. Soichi Takeshita (2005) "Gasification of ASR", Technology for feedstock recycling of plastic wastes, research association for feedstock recycling of plastics, Japan

34. Borgianni C, De Filippis P, Pochetti F, Paolucci M (2001) Gasification process of waste containing PVC. Fuel 81:1827-1833

35. Ma S, Lu J, Gao J (2002) Study of the low temperature pyrolysis of PVC. Energy Fuels 16:338-342

36. Yoshioka T, Akama T, Uchida M, Okuwaki A (2000) Analysis of two stages dehydrochlorination of poly(vinyl chloride) using TGMS. Chem Lett 4:322-323 
37. Kameda T, Fukuda Y, Park K, Grause G, Yoshioka T (2009) Efficient dehalogenation of automobile shredder residue in $\mathrm{NaOH} /$ ethylene glycol using a ball mill. Chemosphere 74:287-292

38. Kameda T, Fukushima S, Shoji C, Grause G, Yoshioka T (2013) Electrodialysis for $\mathrm{NaCl} / \mathrm{EG}$ solution using ion-exchange membranes. J Mater Cycles Waste Manag 15:111-114

39. Kameda T, Shoji C, Fukushima S, Grause G, Yoshioka T (2013) Removal of chloride from ethylene glycol solution using alumina/zeolite membrane as a physical boundary between the organic and aqueous phases. J Mater Cycles Waste Manag. doi:10.1007/s10163-013-0119-8
40. Kamo T, Takaoka K, Otomo J, Takahashi H (2006) Production of hydrogen by steam gasification of dehydrochlorinated poly(vinyl chloride) or activated carbon in the presence of various alkali compounds. J Mater Cycles Waste Manag 8:109-115

41. The proposer was Kanie Propane Co., Ltd. http://www.kaniepropane.co.jp/company.html. Accessed 1 Aug 2013

42. Recycling of Wall Coverings by Shinwa Kankyo Ltd., PVC News (2013) vol 84

43. The topic on September 19th, 2007. http://www.vec.gr.jp/english/ topics/index.html. Accessed 1 Aug 2013 